\title{
Do-It-Yourself Medical Devices: Exploring Their Potential Futures Through Design Fiction
}

\author{
STEAD Michael ${ }^{a^{*}} ;$ COULTON Paul $^{\mathrm{b}}$ and LINDLEY Joseph \\ a HighWire CDT, Lancaster University \\ b Imagination Lancaster, Lancaster University \\ *m.stead1@lancaster.ac.uk \\ doi: 10.21606/dma.2017.475
}

\begin{abstract}
With ever increasing demands on healthcare around the world, ensuring adequate provision for patients is becoming more and more challenging. In this paper, we focus on future healthcare provision, specifically looking at how Do-It-Yourself (DIY) Medical Devices might become widely adopted. Our motivation is to move beyond current debates, which tend to focus on technological capabilities, and instead consider the implications of those technologies for future policy and regulation. Discussions around the future are often challenging, as people find it difficult to envisage how disruptive technologies make futures that stand apart from their current and previous experiences. To facilitate these discussions, we use Design Fiction to speculate about a multi-purpose DIY Medical Device which can support various medical conditions. Using Design Fiction in this way allows us to concretize and explore a future world in which DIY Medical Devices exist, and thus enable meaningful discussions around the social and ethical implications of such DIY medical cultures.
\end{abstract}

DIY Medical Devices; Design Fiction; Making; Design Futures

\section{Introduction}

DIY Healthcare has become a significant topic of discussion in medical and financial forums as they explore the potential of smart and wearable devices to provide greater accessibility to health monitoring and facilitate care in the home (Pang, Zheng, Tian, Kao-Walter, Dubova \& Chen, 2015). One of the drivers for discussions is the proliferation of wearable fitness trackers such as Jawbone and Fitbit which are able to monitor aspects of their environment and their users' lives, display realtime data, and also to share this data with other devices:

In the rest of our lives we're seeing the difference that innovative tech makes, and now the NHS will have a streamlined way of getting ground-breaking and practical new

1. This work is licensed under a Creative Commons Attribution-NonCommercial-Share Alike 4.0 International License. 
technologies into the hands of patients... frontline nurses, doctors and other staff. By doing that, we can transform people's lives. (Stevens, cited in NHS England, 2016, para.11).

Whilst research investigating the use of body-worn technology to collect data has been ongoing since the 1970s (Riphagen, Hout, Krijnen, \& Gootjes, 2013), scholars have recently taken to appropriating Wolf and Kelly's (2010) term 'quantified self' to describe such work. Quantified self research examines all aspects of participants' daily lives including gathering data regards the food they eat, their sleep patterns, changes in mood and biometric information such as blood pressure and heart rate. It has been argued that quantified self tracking not only allows individuals to learn more about themselves, but may also help them take action to become healthier and improve their lives (Lee, 2013). A quantified self approach to DIY Healthcare is thus primarily driven by the value associated with data collection (Dimitrov, 2016), which historically, has been fundamental to improving public health and patient care, whether it was driving sanitary reforms in nineteenth century Europe, or recent quality improvement in surgery (Carrera and Dalton, 2014).

Involving patients in data collection using commercial devices as part of their treatment presents considerable challenges to expected norms, regulations and practices, but the notion of patients developing their own DIY Medical Devices is even more radical. This is not a new idea though; in 1965, Frederick Fascenelli presented 'Electrocardiography by Do-It-Yourself Radiotelemetry' a proposal to allow anyone with basic electronics knowledge to build their own electrocardiogram machine and transmit results to their doctor (Greene, 2016). Although his device never took off primarily due to the complexity of creation and use - Fascenelli was driven by the same desire to improve access to high quality healthcare through technology that we currently see promoted through DIY Healthcare. More recently the interest and discussions around DIY Medical Devices has seen a resurgence through association with the so-called 'Maker Culture'. Maker culture is a grass roots technology centric culture in which participants aim to create new devices, repair and reuse old ones, or to simply tinker. This activity has been enabled by the decreased cost of componentry, increased access to experimental hardware platforms and new forms of fabrication technologies.

The term Democratised Innovation (von Hippel, 2005) is also used to denote practices whereby products and services are developed by the same people who ultimately use them. Within traditional proprietary innovation models, designers and manufacturers exploit internal assets and intelligence to develop standardised, 'closed' products. Contrastingly, when developed with Democratised Innovation principles, knowledge, resources and technologies relating to new products are diffused quickly, efficiently, and more often than not, 'freely' through networks of online and offline communities. This collaborative activity results in products which directly benefit those who created them and frequently also have positive impacts on society at large (von Hippel, 2005).

In the case of DIY Medical Devices, this form of innovation is evident in a variety of emerging activities such as the proliferation of access and availability of 3D printing through 'fab labs' and 'maker spaces' which have provided wearers of prosthetics with new opportunities for designing and modifying their own prostheses (Buehler, Branham, Ali, Chang, Hofmann, Hurst \& Kane, 2015). Demonstrating a DIY mind set, the convergence of the insulin pump with easy and efficient ways of connecting devices to the internet has resulted in insulin-dependent patients, frustrated with their pumps' limitations, sharing their personal continuous-glucose-monitoring data and strategies for augmenting their own devices, through online communities. Perhaps the most notable example of this is Nightscout ${ }^{1}$ which is an open-source platform developed and run by a global community of patients with type- 1 diabetes. The platform combines a CGM (Continuing Glucose Monitor) device which provides constant updates on glucose levels, a DIY data transmitter, and freely available

\footnotetext{
${ }^{1}$ www.nightscout.info
} 
software which enables the CGM data to be shared across throughout the community via cloud data storage (Lee, Hirschfeld \& Wedding, 2016).

Fostering visions of technologies, in particular DIY medical devices, as things that users have a role in producing - as opposed to simply using - is a powerful, egalitarian idea, however, such practices also carry forward risks associated with individuals taking technologies on which their life depends, into their own hands. Regulation pertaining to the production of medical devices is in place to prevent risk to patients from equipment that has not undergone a rigorous approval process. Currently in the UK, Medical Devices are classified under European regulations (European Commission, 2017) before undergoing a certification assessment relative to the class of device. Depending on its intended purpose, a medical device may be classified within Class I, Ila, IIb or III, with Class III covering the highest risk products (GOV.UK, 2017). The higher the classification, the greater the level of assessment required. Classification of a medical device will depend upon several factors including:

- how long the device is intended to be in continuous use;

- whether or not the device is invasive or surgically invasive;

- whether the device is implantable or active;

- whether or not the device contains a substance, which in its own right is considered to be a medicinal substance and has action ancillary to that of the device (Halliday, Kutty \& Rakos, 2017).

Classification is primarily the first step towards conformity assessment and obtaining the CE mark (a logo placed on medical devices to denote that they conform to the requirements in the regulations). The CE mark shows that the device is fit for its intended stated purpose and that it meets legislation designed to ensure patient safety. Further, such approval signifies that a product can be freely marketed and sold anywhere within the European Union. In the UK, this activity is overseen by the Medicines and Healthcare Products Regulatory Agency (MHRA) which is responsible for regulating medicines, medical devices and blood components for transfusion in the UK. ${ }^{2}$

In the context of Democratised Innovation, fulfilling these classification requirements can be prohibitively expensive and hence severely restricts the participation of those with the technical skills to create or modify their own devices, and subsequently stifles community growth. If we are to move beyond this situation we need to first conceive a future which accommodates the potential for future DIY Medical Devices to be fully exploited. However, getting authorities to engage with futures is often difficult as they can get bogged down within discussions of the present, that are, in turn, more often than not based upon the past (Gonzatto, van Amstel, Merkle \& Hartmann, 2013) In this paper, we use Design Fiction to explicate a future in which the widespread utilisation of DIY Medical devices plausibly exists. Although the design fiction methodology is becoming increasingly well established, one of its foundational tenets is the power of the 'diegetic prototype', a concept whose foundations include the realisation that fictional representations of medical devices can have a profound influence over the publics' perception of real medical devices (Kirby, 2010).

\section{What is Design Fiction?}

Design Fiction sits within a range of design practices known as speculative design which focus on the values embodied within a particular design artefact rather than the intended use of the artefact. The main attributes that these speculative practices share is that they are: free from the commercial constraints that might limit the design process; use prototypes as the main method of enquiry; present these prototypes within fictional alternate past, presents, or futures; and, often exhibit an irreverent or playful quality as a means of engaging the audience (Coulton, Burnett \& Gradinar, 2016). These practices enable designers to not only to question how things might be, but to also unpack why things are, the way they are. Design Fiction is a particular form of speculative design

\footnotetext{
${ }^{2}$ www.gov.uk/government/organisations/medicines-and-healthcare-products-regulatory-agency
} 
which prototypes potential futures where emerging technologies have been widely adopted in order to understand what the potential broader ethical and societal implications of those technologies might be (Lindley, Coulton \& Sturdee, 2017).

As a result of Design Fiction still being an emerging field, there remains a number of conflicting definitions of what it is and what it is for. To make our position clear within this research, we consider Design Fiction as a world building activity. Whilst the critical intentionality of Design Fiction distinguishes it from similar corporate endeavours which present futures commensurate with values of the corporation (Coulton, Lindley, Sturdee \& Stead, 2017). the means of Design Fiction (the objects and artefacts produced by practice) are diverse and varied, yet the primary aim of Design Fiction is always the creation of a fictional world:

Design Fictions are collections of artefacts, that, when viewed together build a fictional world. The artificially built world is a prototyping platform for the very designs that define it, meanwhile those designs reciprocate in kind and prototype the world. (Coulton et al, 2017, p. 15).

We propose two useful metaphors for considering how the individual artefacts relate to the world. Firstly, let us imagine a Design Fiction world as a distinct entity, one that we can see the overall shape of, but whose complex internal structure is hidden from view. What is in view, however, are a series of entry points. As shown in Figure 1, each artefact that contributes to the creation of the Design Fiction also plays its role as a metaphorical entry point to the fictional world.

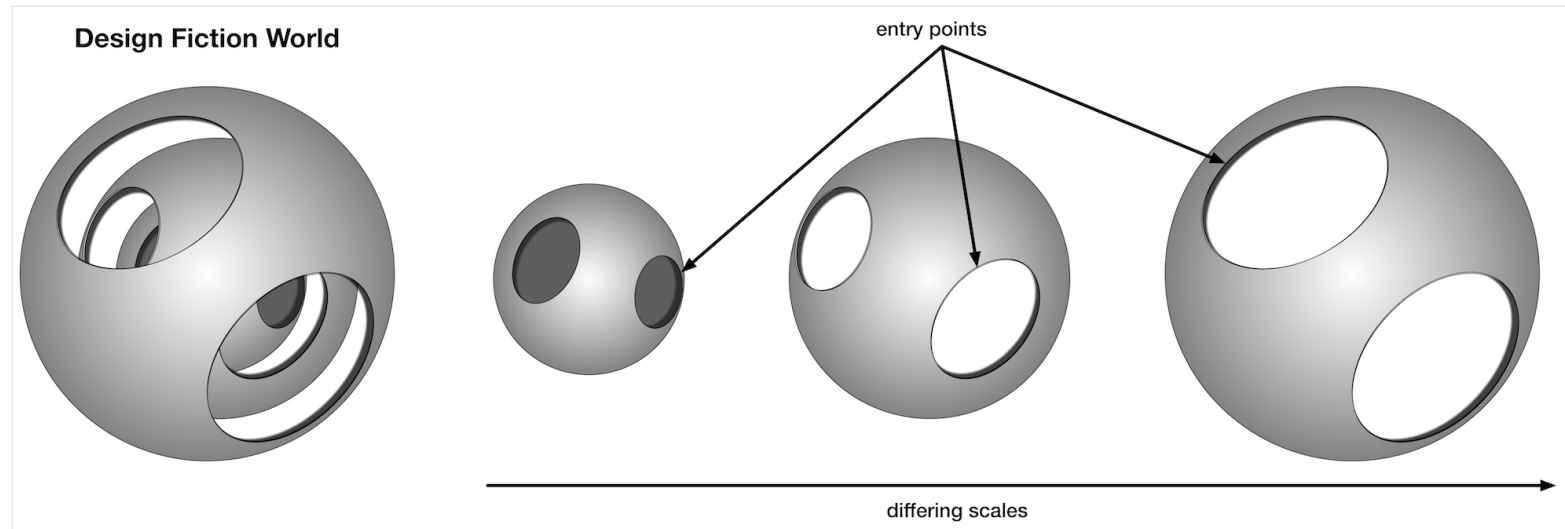

Figure 1 Design Fiction as World Building

The second metaphor, which works in unison with the first, is inspired by Charles and Ray Eames' iconic film about the relative size of things in the Universe, Powers of 10. The film shows our world from number of frames of reference (literally drawn as squares in the film) starting with a 1 meter squared section of an image that includes a couple sitting having a picnic. The camera then zooms out and increases the visible area by one power of 10 every 10 seconds. This changing scale is a device that encourages the viewer to constantly reconsider the scene being viewed. It is important to note that our use of such framing is not to suggest that Design Fictions adhere to the configuration 1 power of 10 per 10 second. Rather, we contend that the basic concept of shifting scale can be applied to the consideration of Design Fiction worlds and the artefacts that create them. We can think of each individual artefact that constructs the world as a representation of that world, but at a different scale (Coulton et al, 2017).

In the following section, we describe a Design Fiction as both a means to illustrate how Design Fictions are created, as well as to present a future world in which a DIY medical device might plausibly exist. Through the process of envisioning, designing and building this Design Fiction world, 
we create a reciprocal prototyping relationship whereby the designs create and test the world, and the world tests the prototype designs.

\section{Do-It-Yourself Medical Device Design Fiction}

Using the aforementioned concept of Design Fiction as world building, we present HealthBand. This Design Fiction uses a range of artefacts as entry points to its world and to represent that world at different scales. Each artefact also has a different focus, with the aim of facilitating multiple different 'readings' or interpretations of this future world. As the principle aim for Design Fictions is to enable, rather than shutdown, a wide a range of discussions as possible, these artefacts are presented in forms that are likely to be recognisable in relation to their audience's current experience. The aim is not to present the future as fantastical but rather as mundane, for it is through this mundanity that the audience's own lived experiences may come into relief. Further, such framing helps to realistically situate the artefacts within a plausible near future (Coulton et al, 2017). This mundanity gives Design Fictions a distinctly 'everyday quality', which in turn places our work in clear contrast to other speculative or critical design practices, whose artefacts are frequently intended for exhibition in galleries (Auger, 2013). In the following paragraphs, we design and present the artefacts created as part of the HealthBand Design Fiction.

\subsection{Legislation}

As previously discussed, present legislation overseen by the MHRA would prohibit the use of DIY Medical Devices such as HeathBand in a clinical setting unless these products can be proven to meet all the current regulation. Interestingly however, whilst the guidelines explicitly state that CE marks cannot be obtained for custom made health devices as they "must still meet the requirements in the directives and the type of device should be labelled clearly" (MHRA, 2016, para. 21), they also suggest it is not completely out of the question:

You don't need to get these checked by a third party to show they conform with the requirements but you need to draw up a statement to declare their compliance for custom-made devices, clinical investigations and performance evaluation devices. (MHRA, 2016, para. 22).

This means that a change in the law, rather than a completely new law, would be a necessary component of any plausibly wide adoption of such a technology. In order to highlight this point, Figure 2 presents an extract from a fictional white paper. In the UK, white papers are policy documents produced by the Government that set out their proposals for future legislation. White papers may include a draft version of a Bill that is being planned to change existing law or introduce new legislation. This provides a basis for further consultation and discussion with interested or affected groups, and allows final changes to be made before a Bill is formally presented to Parliament. As white papers are aimed at facilitating discussions about the future, it would arguably make it the most appropriate artefact to engage those who are able to facilitate the changes in legislation required for DIY Healthcare and Medical Devices. As a white paper is particular to the UK, it would not necessarily make sense in the context of another country and thus highlights how the forms of a particular Design Fiction need to be chosen relative to their intended audience (Coulton, Lindley \& Akmal, 2016).

\subsection{Crownfunding Campaign}

As part of the HealthBand Design Fiction we wished to address the question of how might the development of such devices be funded if the expectation is that it would effectively exist outside current commercial models for medical device production. Inspired by the way in which many Internet of Things (IOT) products services are being financed, we propose that such DIY wearables would likely be crowdfunded. Healthcare wearables are a popular trope of the IoT, thus appropriating the crowdfunding model lends plausibility to the Design Fiction, particularly if the audience is familiar with developments in loT. This fictional frame is illustrated in Figure 3 which 


\section{The case for change}

1.1 Rising aging populations living with chronic health conditions like diabetes, dementia and Parkinson's disease have put an incessant strain on the NDHS. Although it has been proven that these conditions can be successfully managed by patients using wearable health devices, due to abstruse health product legislation, too few devices have been made available to patients over the last decade. At the end of January 2027, 345 of the 418 local health authorities had put forward 'autonomous patient digital health' policies for consultation. Since then, the National Digital Health Framework has also published its report on "homemade' wearable health devices ${ }^{20}$ as a means to fulfil the terms of service pledged by the NDHS in 2021.

1.2 Changes to digital health services have remained slow, expensive and bureacratic, with arguments about how many patients will be able to manage their own healthcare autonomously and what level of services are offered
1.4 In response, this chapter sets out our proposals to reform health product legislation as well as identifying sufficient funding and expertise to make the most of the proposed changes; with community involvement to make the best outcomes for both 'autonomous citizens' and those continuing to use limited health services. 1.5 A number of the proposals build on consultantions and reviews conducted over the last year: the report of the Local Health Device Group; consultations on changes to the National Digital Health Framework, ${ }^{21}$ frontline service reviews (the results of patient-led care trials at different sites across the country); and the National Patient Wearable Review also provided evidence. ${ }^{22}$ The Government has taken account of responses to these consultations in deciding the way forward. A summary of the responses to each consultation is being published alongside this White Paper.

\section{Getting tech in place}

Making sure every UK citizen has arroce to dinital torhnnlnniec and

Figure 2 UK White Paper proposing changes in legislation that would allow DIY Medical Devices

\section{FINDING FUNDING}

Phil \& I uploaded our prototype to the crowdfunding site LightBulb. We were blown away by the response...

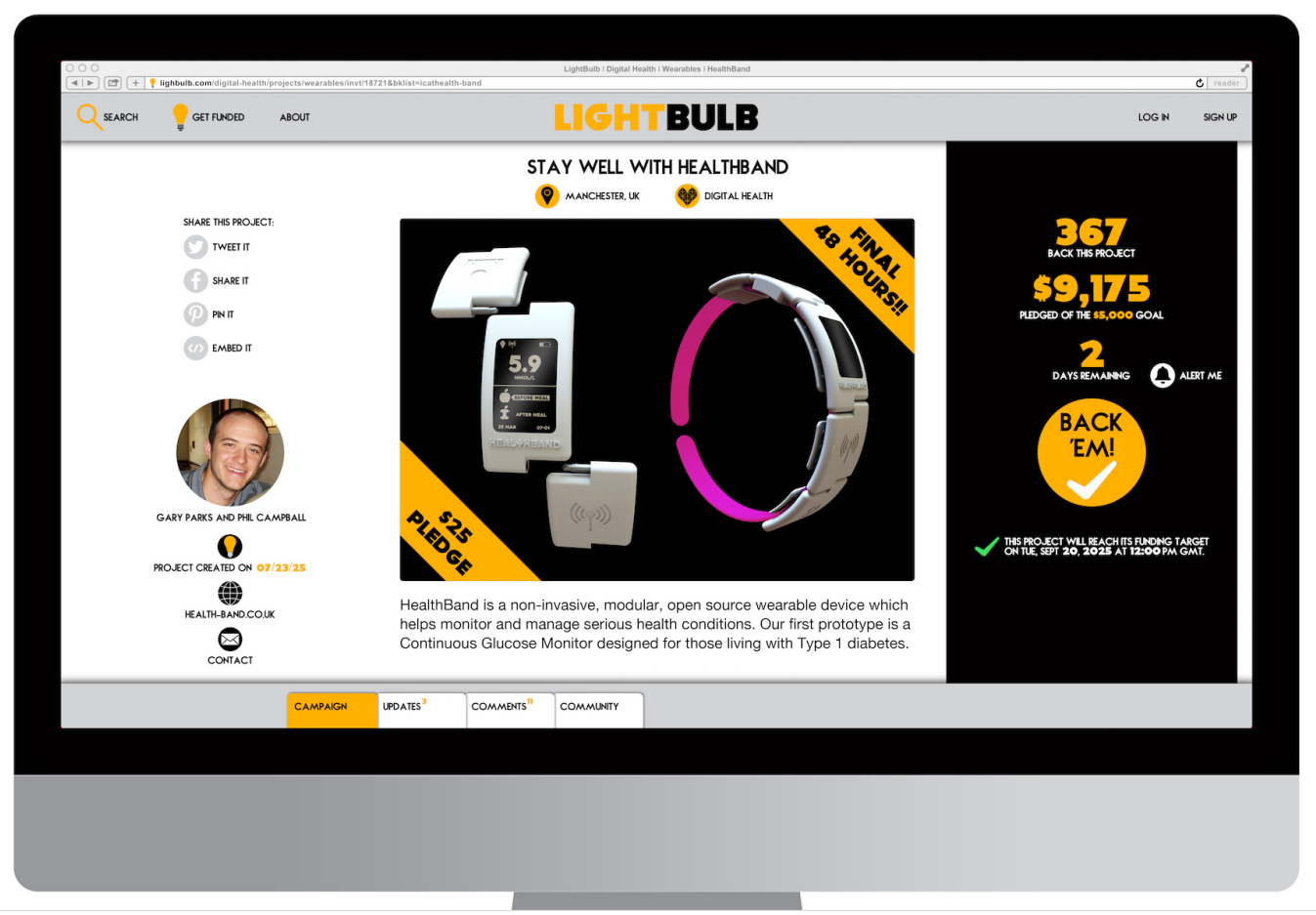

Figure 3 HealthBand Crowdfunding Campaign 
explains the origins of and motivations behind the product and discusses the success of a crowdfunding campaign which funded the production of the first three HealthBand prototypes.

The artefacts also detail how people can become actively involved in developing the project further by donating funds, creating new modules based on an open design template, or simply purchasing the device. Note, that whilst we created a fictional crowdfunding site Lightbulb for this fiction, in other work we have used existing organisations such as Kickstarter within the fictions as there is a strong argument that all the products and services that appear on these sites are indeed 'fictional' until they are successful in reaching their funding goal and the product and/or services are delivered to contributors.

\subsection{Developer Stories}

To illustrate the how and why it was created, each of the three HealthBand prototypes is presented in more detail in Figure 4. In the first developer story, Gary and Phil from Manchester in the UK, describe their original motivation for initiating the HealthBand project - to aid their cousin who has Type 1 diabetes - and the ensuing success of their crowdfunding campaign. Their story argues for the importance of personalisation, hence proposing a modular design, which encourages others to innovate on the HealthBand platform. This particular component of the design fiction draws inspiration from Nightscout, in that it focuses on self-monitoring of diabetes symptoms. It also seeks to extend the concept such that medical devices themselves become open-source hardware platforms.

The second story concerns Alicia from the USA who, having been excited by seeing the original diabetes monitor, decided to create memory aid and tracker modules. Alicia was inspired to design
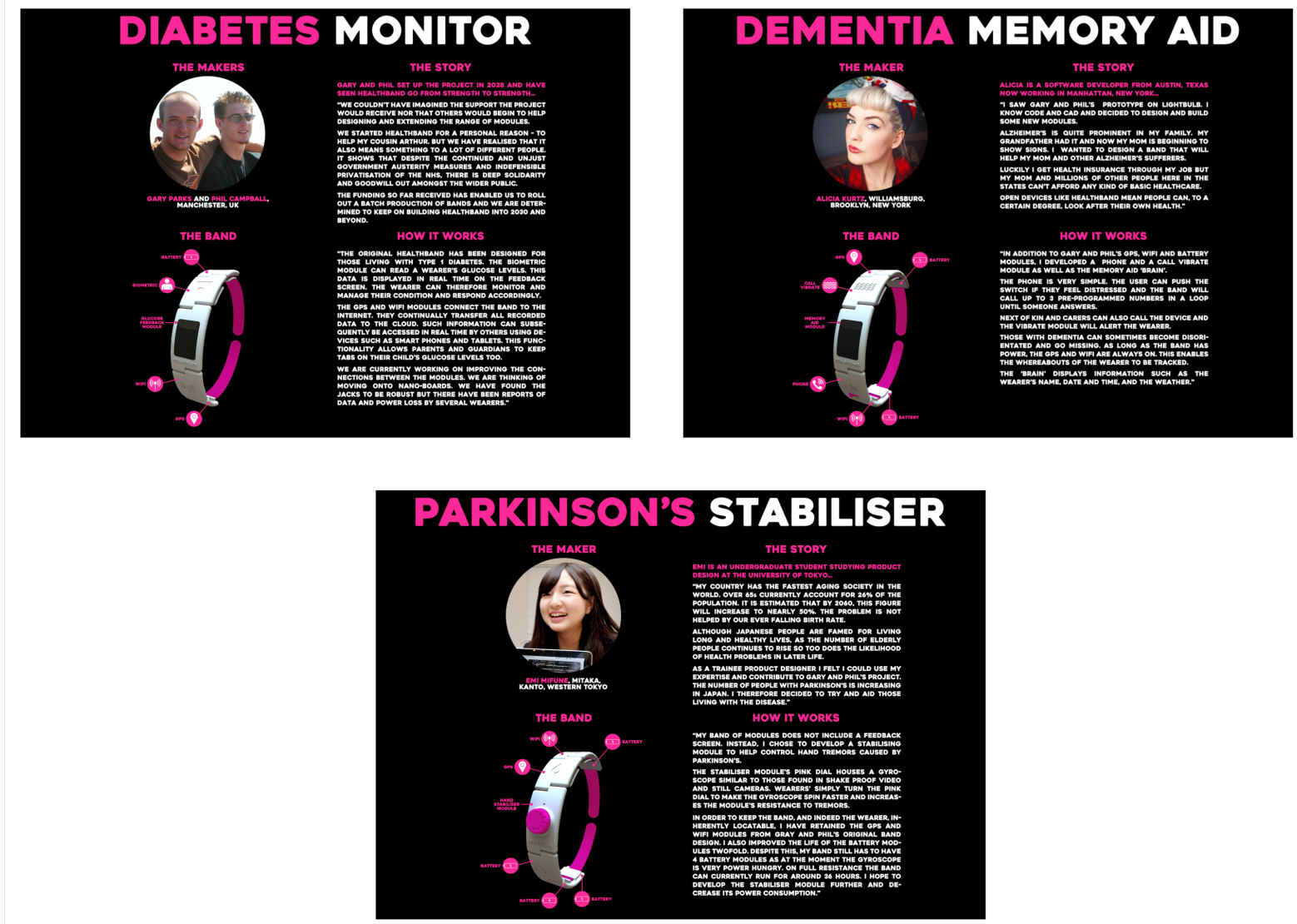

Figure 4 HealthBand Developer Stories 
the memory aid module both due to the prevalence of Alzheimer's in her family, and a in response to the difficulties faced by many in the USA of obtaining health insurance. In terms of the Design Fiction it draws from health reports from the Alzheimer's Society ${ }^{3}$ who highlight that Alzheimer's is the most common cause of dementia, affecting 62 per cent of those diagnosed with the syndrome. There are currently 850,000 people with dementia in the UK, with numbers set to rise to over 1 million by 2025 and are further expected to soar to 2 million by 2051. Introducing the USA perspective not only highlights that Dementia is a global issue, but also emphasises that individual countries have particular problems with access to healthcare, in this case access to affordable, comprehensive medical insurance in the USA.

The final story features Emi from Japan which highlights the issue of their increasingly aged society. Indeed, Japan's population is expected to see the number of over 65 s to grow to nearly $50 \%$ by 2060 (McCurry, 2016) while also experiencing a declining birth rate (Soble, 2017). In this story, Emi has developed HealthBand modules which are specifically designed to stabilise hand tremors which are a common symptom of Parkinson's disease. We contend that his story, in part highlights that symptoms exhibited by patients vary from individual to individual which in turn emphasises the need for a flexible and reconfigurable design solution.

\subsection{Modular Design}

As previously discussed, individual patients' needs and symptoms can be quite varied, particularly when treating complex conditions such as dementia which unfortunately often develops alongside a range of other challenging health issues. Further, as dementia is a degenerative illness, the needs of a particular patient will vary over time. Technological solutions should seek to address different aspects of a condition and the platform should therefore be flexible enough to allow devices to be configured and reconfigured in order to meet the dynamic needs of users.

Figure 5 depicts an exploded view of the HealthBand prototype and illustrates how each of its modules connect together via $3.5 \mathrm{~mm}$ jacks. The modules each have a rear 'clip' which must be used to secure them to a fuchsia 'Snap-On' wristband. In the fiction, the band is said to be comprised of a flexible metal strip coated in a layer of durable but soft to touch silicone. This feature means that the design is versatile, in that it would be able to fit a wide variety of wrist sizes.
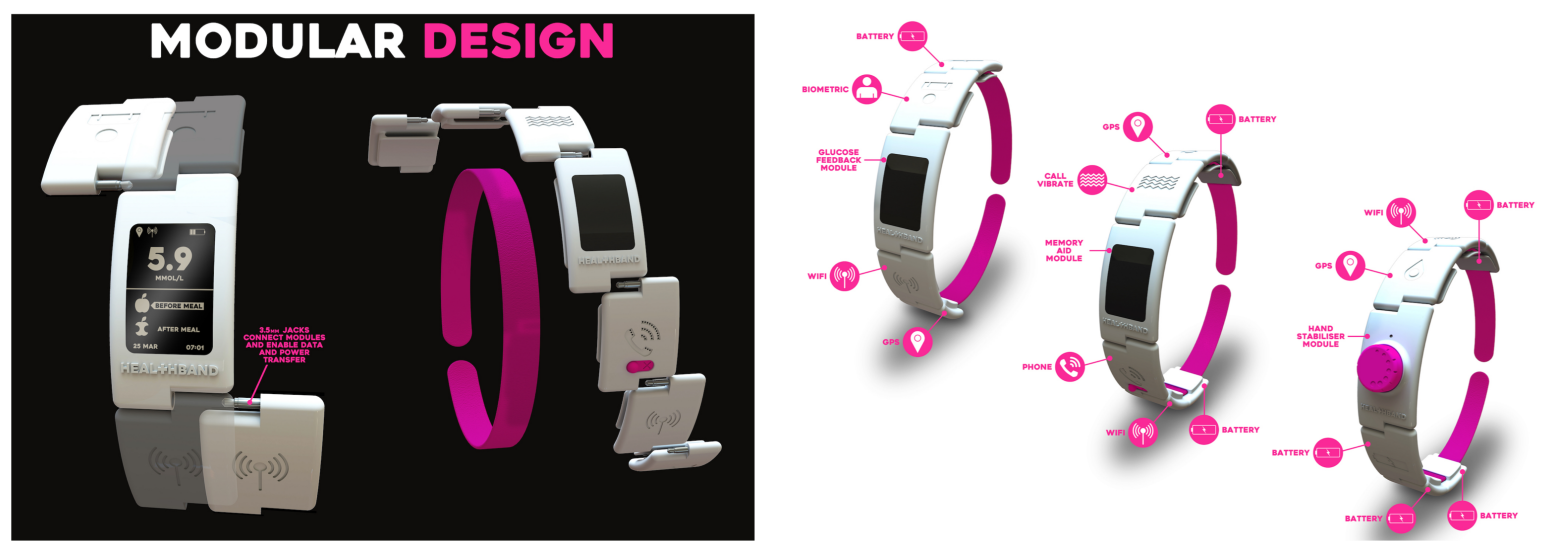

Figure 5 HealthBand modular design

${ }^{3}$ www.alzheimers.org.uk 
The modularity of the HealthBand prototype was partly inspired by the Blocks modular smart watch which was first developed during the Intel Make It Wearable Challenge 2013. After being selected as one of the finalists and receiving $\$ 50,000$ funding from Intel, the team behind the product then sought further capital via the Kickstarter crowdfunding platform (Charara, 2016). Despite numerous release dates being announced since early 2016, the device has yet to be released with the current estimated date being the $1^{\text {st }}$ quarter of 2018. Given the continual delays, there is possibility that Blocks may suffer the fate of ultimately becoming vapourware (Coulton and Lindley, 2017).

\subsection{Fabrication Permit}

The example of Blocks is quite representative of the current volatility across development of the loT, in that it is a constantly evolving landscape with devices, services and companies entering and leaving the market at a rapid rate. Whilst this precariousness may be acceptable for early adopters of these technologies, it is unlikely to instil confidence in those responsible for healthcare provision. This suggests that while we may wish to take advantage of Democratised Innovation in the creation of such devices, there is likely a need to consider how we can facilitate trust in those creating such devices and ensure some level of accountability amongst device developers, health service providers and legislators. Such a task is of course highly complex and whilst we are not realistically suggesting this as a solution as part of the Design Fiction, to begin considering notions of accountability, we have created a device fabrication permit.

The permit draws upon present day medical device certification processes (MHRA, 2015) and also introduces the notion of linking a particular condition to the permit in a similar vein to how devices are currently classified. In this way, the risk to patients can be handled in a more nuanced manner and links directly to a developer's experience, as opposed to a simple, blanket legislation which might grant universal permission to fabricate medical devices.

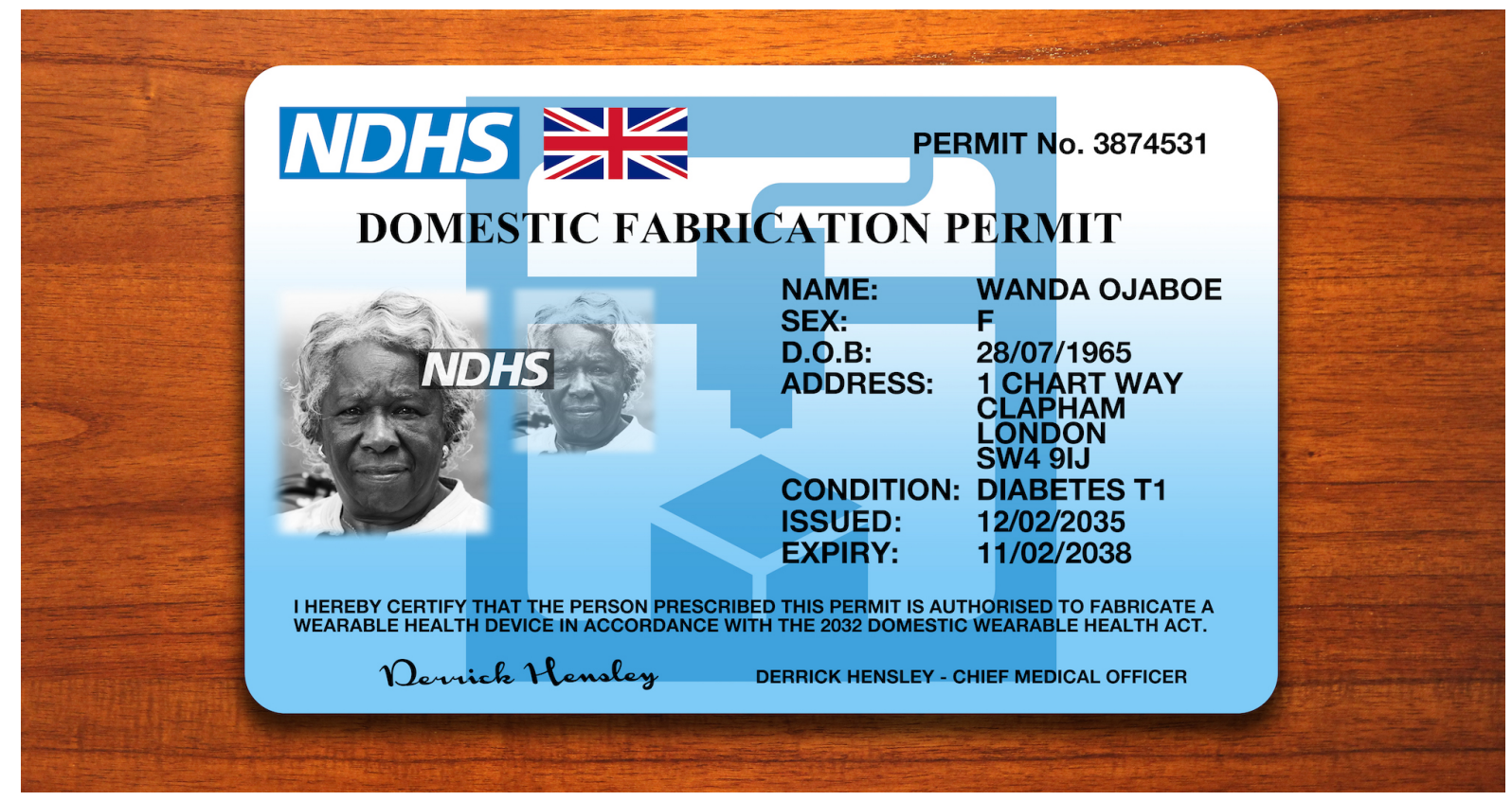

Figure 6 Domestic Fabrication Permit

\subsection{Guide to Obtaining Device Certification}

In proposing something as radical as DIY Medical Devices we are aware that the experience of healthcare professionals would become profoundly different. It is crucial that nurses, doctors, and 
other allied health professionals have confidence in the widespread use of such devices and the health-critical data that they collect:

While many healthcare wearables can be positive feedback tools and motivational aids, doctors ultimately want clinically proven products whose data they can use to make clinical decisions (cited in Wall, 2016, para.22).

With this in mind, the last artefact we present for the Design Fiction is a pamphlet (Figure 7) which highlights a set of actions which device developers need to undertake before their device might be considered and put forward for clinical trials. Whilst the pamphlet follows similar requirements currently defined by the MHRA, it also introduces new requirements such as ensuring the software and hardware are open for both modification, the need for any data to be handled in a secure and ethical manner, and that devices would be considered by a specialised professional service before it could move on to the next stage of certification. As with the fabrication permit, this is not being offered as the solution to certification but rather providing a starting point for deliberations on what the actual requirements of a new certification process might be.
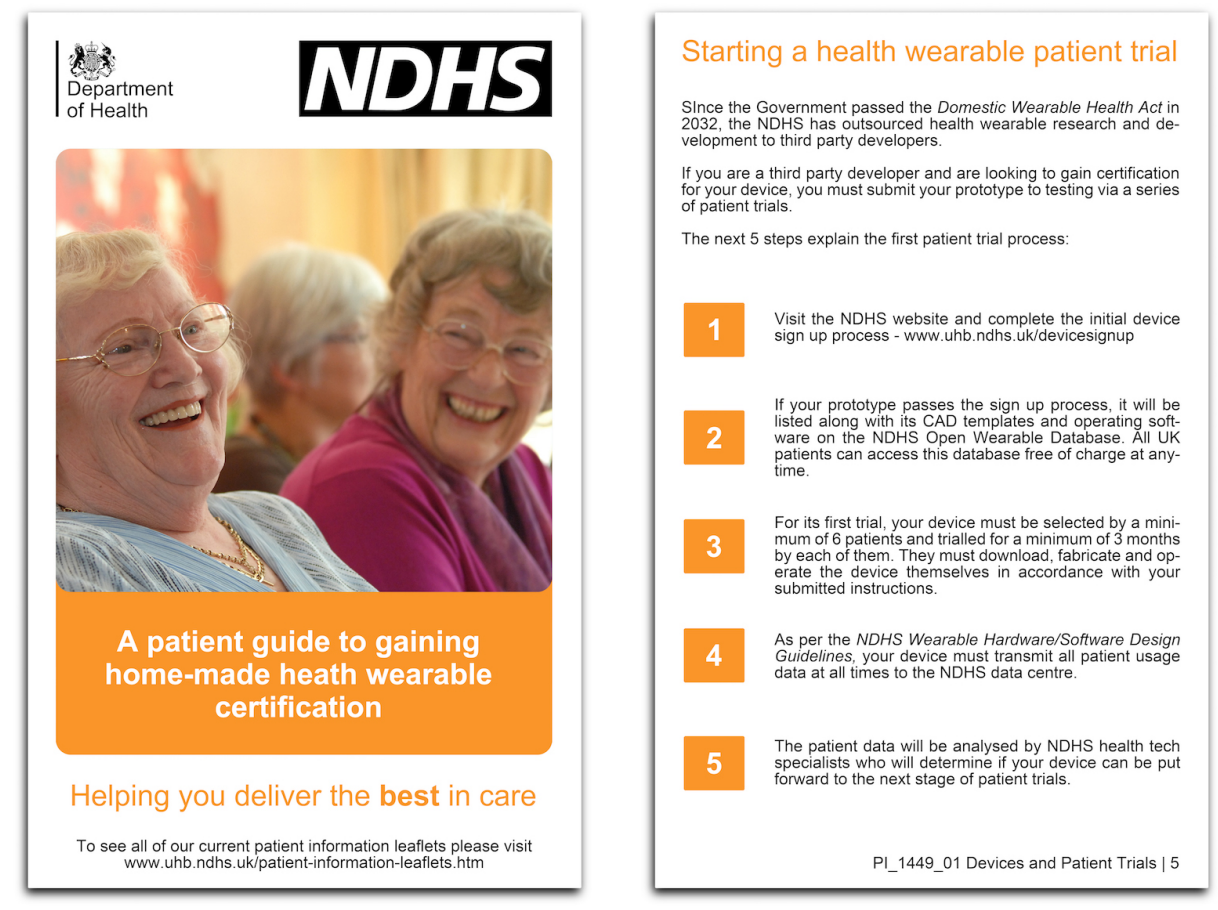

Figure 7 Device Certification Guide

\section{Reflections on HealthBand World Building}

What is evident when creating Design Fictions is that whilst the artefacts are fictional, if they are to facilitate meaningful questions around the futures they portray, they need to be conceived with the same commitment to detail as if they were actually being designed and produced. Further, we argue that it is the world building approach which allows such detail to be developed and which can address some of the associated complexity which emerges during the transition from an emerging technology with interesting potential to one capable of reaching widespread adoption in a variety of sectors. Crucially, the aim of the Design Fiction is not to present solutions to this complexity but 
rather to ensure that there is discourse that considers what factors may need to be addressed during this transition. Although the design process of creating the HealthBand Design Fiction has been discussed in this paper, we have, as yet, only had preliminary discussions with healthcare professionals, patients and carers with experience of the relevant conditions. It is imperative therefore to further reflect on these artefacts in terms of how they might extend to particular discussions.

Initially we considered that legislation would be the most contentious factor in enabling DIY medical devices, however, healthcare is an area that undergoes constant innovation with regards to medicines and devices. The challenge is thus: how can Democratised Innovation be facilitated within the existing frameworks which have been set-up to mitigate risk. The artefact itself implies that these risks can be overcome and establishes a case for change but how such risks could be mitigated and how liability may be addressed are not explored in any depth. We posit that these issues could perhaps be addressed to a further degree within the fiction by presenting potential opponents to the envisioned changes to legislation allowing DIY Medical Devices. Such opposition might take the form of reports from health policy think tanks, healthcare practitioners concerned with risks to public safety, medical ethics committees and competitors, that is, corporate medical device manufacturers, who currently must spend large sums of capital to develop medical devices which meet stringent design/production regulations. Legislatorial reform may also lead to a reduction in manufacturers' share of the medical device market and subsequently reduce their ability to increase profits.

When we created the crowdfunding campaign for HealthBand, it was primarily an artefact through which we chose to emphasise the patient initiated innovation and provide a frame in which the individual developer stories could highlight the factors leading people to advocate for DIY Healthcare and DIY Medical Devices. In many respects to those familiar with crowdfunding and creating IoT devices, here the fiction perhaps lacks detail that might produce more meaningful discussions. For example, what would be a realistic funding goal to achieve the creation of a new device? What exactly would users get for their investment? Given that a number of crowdfunded loT devices have obtained funding but not met initial delivery targets, or drifted towards vapourware, would the ethical requirements placed on those developing DIY Medical devices through these crowd funding platforms need to be different from those say developing products and services for the home entertainment market?

In terms of the modular design of the HealthBand, this aligns strongly with the current emphasis on providing greater focus on the needs of individual patients rather than particular conditions and how these needs are likely change over time. This modular aspect is also particularly useful for extending the scope of the more fictional DIY Medical Device world to include other conditions which may present very different challenges than those currently envisioned.

The permit is primarily a means of linking current medical device certification with potential ways of how this might be adapted to allow Democratised Innovation and medical device production on a more individual level. There are subsequent challenges that the permit leaves unanswered, for example, how is the permit obtained and what are the requirements for applicants regards fabrication qualifications, liability, insurance etc. As it is a fabrication rather than developer permit, it also suggests that designs might be outsourced to certified individuals, or even machines, to be built thus allowing innovations to be disseminated through open-source practices. As with other previously discussed artefacts, it primarily presents a positive perspective and other, more negative questions might relate to whether a black market for permits and devices might emerge and how such actions might be addressed.

The device trials pamphlet introduces the requirement for clinicians to have confidence that the devices have proven benefit that would draw from, rather than be separate to, existing practices. This might be a useful starting point for discussions with those involved in medical trials as to how 
such practices might potentially operate. Such insights could then be used to initiate future iterations of, or additions to, the fictional world depicted by this design fiction. Further evolution of this work may result in a dynamic prototype usable by policy makers, community-based makers, patients, and technology developers to understand the safety challenges around widespread adoption of DIY Medical Devices, as well as the economic and health centric opportunities.

\section{Conclusions}

Whilst DIY Medical Devices are garnering considerable attention in the media, academia and industry, they are drawing from a design-maker culture that is challenging current manufacturing practices which are often less complex than the practices relating to the production of real medical devices. Presently the fiction only explores one example of a possible future - that of the three developers who we can describe as 'lay users'. Envisioning how healthcare professionals might become involved with the HealthBand concept could no doubt provide other interesting points of entry into the fictional world. For example, might doctors and nurses also begin to fabricate DIY products to cater for specific patient needs? Indeed, if we wish to progress the ideas initiated by the HealthBand fiction beyond their exciting potentiality, we need to further explore the implications of DIY Medical Devices from multiple perspectives and highlight the issues that would need to be addressed if they are to develop to a point of widespread adoption. Crucially, the fiction is built upon our own - the authors' - subjective interpretations regards the subject. While it is beyond the scope of this paper, real-world evaluations and interpretations regards HealthBand by a range of key stakeholders such as patients, healthcare professionals, regulators and medical device designers may well provide a rich source of insights which can in turn be used to instil the fiction with more rigour and criticality.

Despite the fiction's outlined limitations, we argue that Design Fiction is a highly useful way to address the implications for adoption of DIY medical devices as it is a speculative design practice specifically aimed at engaging with such a challenge. The paper illustrates the process of envisioning, designing and building a Design Fiction world - a reciprocal prototyping relationship whereby the designs create and test the world, and the world tests the prototype designs. We believe this creative practice has much to offer those considering the futures of emerging technologies, in particular for the healthcare sector, or other sectors that must make similarly complex and safetyconscious judgements.

Acknowledgements: This work is funded by the Digital Economy programme (RCUK Grant EP/G037582/1) supporting the HighWire Centre for Doctoral Training. (http://highwire.lancs.ac.uk) and the PETRAS Cyber Security Hub for the Internet of Things (RCUK Grant EP/N02334X/1).

\section{References}

Auger, J. (2013). Speculative design: crafting the speculation. Digital Creativity, 24(1), 11-35. doi:10.1080/1462 6268.2013 .767276

Buehler, E., Branham, S., Ali, A., Chang, J. J., Hofmann, M. K., Hurst, A., \& Kane, S. K. (2015). Sharing is caring: assistive technology designs on thingiverse. $\mathrm{CHI} 15$ Proceedings of the $2015 \mathrm{CHI}$ Conference on Human Factors in Computing Systems (pp. 525-534). ACM. doi:10.1145/2702123.2702525

Carrera, P. M., \& Dalton, A. R. (2014). Do-it-yourself healthcare: the current landscape, prospects and consequences. Maturities, 77(1), 37-40. doi:10.1016/j.maturitas.2013.10.022 
Charara, S. (2016, 23 Oct 2017). Blocks Modular Smartwatch Is Delayed (Again) Until December 2016. Wareable. Retrieved from http://www.wareable.com/smartwatches/blocks-price-specs-release-datefeatures-2016

Coulton, P., Burnett, D., \& Gardiner, A. I. (2016). Games as speculative design: allowing players to consider alternate presents and plausible futures. In P. Lloyd, \& E. Bohemia (Eds.), Proceedings of Design Research Society Conference 2016. (pp. 1609-1626). (Proceedings of DRS 2016; Vol. 1). Design Research Society. doi: 10.21606/drs.2016.15.

Coulton, P., Lindley, J., \& Akmal, H. A. (2016). Design fiction: does the search for plausibility lead to deception? In P. Lloyd, \& E. Bohemia (Eds.), Proceedings of Design Research Society Conference 2016. (pp. 369-384). (Proceedings of DRS 2016; Vol. 1). Design Research Society. doi: 10.21606/drs.2016.148

Coulton, P., \& Lindley, J. G. (2017). Vapourworlds and design fiction: the role of intentionality . The Design Journal, 20(Suppl. 1), S4632-s4642. doi: 10.1080/14606925.2017.1352960

Coulton, P., Lindley, J. G., Sturdee, M., \& Stead, M. (2017). Design fiction as world building. In Proceedings of Research through Design Conference 2017. (pp. 163-179).

Dimitrov, D. V. (2016). Medical internet of things and big data in healthcare. Healthcare informatics research, 22(3), 156-163. doi:10.4258/hir.2016.22.3.156

European Commission (2017, 05 Mar 2018). REGULATION (EU) 2017/745 OF THE EUROPEAN PARLIAMENT AND OF THE COUNCIL of 5 April 2017 on medical devices, amending Directive 2001/83/EC, Regulation (EC) No 178/2002 and Regulation (EC) No 1223/2009 and repealing Council Directives 90/385/EEC and 93/42/EEC. Retrieved from http://eur-lex.europa.eu/legal content/EN/TXT/?uri=uriserv:OJ.L_.2017.117.01. 0001.01.ENG\&toc=OJ:L:2017:117:TOC

Frayling, C. (1993). Research in Art and Design, Royal College of Art, Research Papers 1:1.

Gaver, W. (2012). What should we expect from research through design? CHI '12 Proceedings of the $2012 \mathrm{CHI}$ Conference on Human Factors in Computing Systems (pp. 937-946). ACM. doi:10.1145/2207676.2208538

Greene, J. A. (2016). Do-it-yourself medical devices-technology and empowerment in American health care. New England Journal of Medicine, 374(4), 305-308. doi:10.1056/NEJMp1511363

Gonzatto, R. F., van Amstel, F. M. C., Merkle, L. E., \& Hartmann, T. (2013). The ideology of the future in design fictions. Digital Creativity, 24(1), 36-45. doi:10.1080/14626268.2013.772524

GOV.UK. (2017, 05 Mar 2018). MHRA - An introductory guide to the medical device regulation (MDR) and the in vitro diagnostic medical device regulation (IVDR). GOV.UK. Retrieved from https://www.gov.uk/ government/uploads/system/uploads/attachment_data/file/640404/MDR_IVDR_guidance_Print_13.pdf

Halliday, S., Kutty, J., \& Rakos, R. (2017, 05 Mar 2018). MDR - Device Classification - Conformity Assessment Safety \& Performance Requirements - Technical Documentation. BSI. https://www.bsigroup.com/meddev /LocalFiles/en-US/roadshow-resources-2017-fall/bsi-mdr-classification-conformity-sprs-documentation.pdf

Kirby, D. (2010). The Future is now: diegetic prototypes and the role of popular films in generating real-world technological development. Social Studies of Science, 40(1), 41-70. doi:10.1177/0306312709338325

Lindley, J. G., Coulton, P., \& Sturdee, M. (2017). Implications for adoption. CHI '17 Proceedings of the $2017 \mathrm{CHI}$ Conference on Human Factors in Computing Systems. (pp. 265-277). ACM. doi: 10.1145/3025453.30257 42

Lee, V. R. (2013). The Quantified Self (QS) Movement and Some Emerging Opportunities for the Educational Technology Field. Educational Technology, (November-December 2013), 39-42. https://digitalcommons.usu .edu/itls_facpub/480 
Lee, J. M., Hirschfeld, E., \& Wedding, J. (2016). A patient-designed do-it-yourself mobile technology system for diabetes: promise and challenges for a new era in medicine. Jama, 315(14), 1447-1448. doi:10.1001/jama.2 016.1903

McCurry, J. (2016, 23 Oct 2017). Japan Running Low on Workers as Proportion of Over-65s Hits Record Levels. Guardian Online. Retrieved from https://www.theguardian.com/world/2016/jun/30/japan-census-over65s-record-27-population-immigration

MHRA, (2015, 23 Oct 2017). Conformity Assessment Routes. GOV.UK. Retrieved from www.gov.uk/governmen t/uploads/system/uploads/attachment_data/file/476902/Conformity_assessment_routes_chart_Nov15.pd $f$

MHRA, (2016, 23 Oct 2017). Medical Devices: Conformity Assessment and the CE Mark. GOV.UK. Retrieved from https://www.gov.uk/guidance/medical-devices-conformity-assessment-and-the-ce-mark

NHS England. (2016, 23 Oct 2017). NHS Chief Launches New Fast Track Funding so NHS Patients get Treatment Innovations Faster. NHS. Retrieved from https://www.england.nhs.uk/2016/06/treatment-innovations/

Pang, Z., Zheng, L., Tian, J., Kao-Walter, S., Dubrova, E., \& Chen, Q. (2015). Design of a terminal solution for integration of in-home health care devices and services towards the Internet-of-Things. Enterprise Information Systems, 9(1), 86-116. doi:10.1080/17517575.2013.776118

Riphagen, M., Hout, M. V., Krijnen, D., \& Gootjes, G. (2013). Supporting reflective learning for daily activities using an interactive dashboard. Proceedings of the 6th International Conference of Education, Research and Innovation, ICERI 2013 Seville (pp. 3239-3249).

Soble, J. (2017, 23 Oct 2017). Japan's Falling Birth Rate Posing Serious Problems for Economy. Independent Online. Retrieved from http://www.independent.co.uk/news/world/asia/japans-falling-birth-rate-posingserious-problems-for-economy-a7770596.html

von Hippel, E. (2005). Democratizing Innovation. Cambridge: MIT Press.

Wall, M. (2016, 23 Oct 2017). The Proven Health Trackers Saving Thousands of Lives. BBC. Retrieved from http://www.bbc.co.uk/news/business-37972606

Wolf, G. (2010, 23 Oct 2017). Know Thyself: Tracking Every Facet of Life, from Sleep to Mood to Pain, 24/7/365. Wired. Retrieved from https://www.wired.com/2009/06/Ibnp-knowthyself/

About the Authors:

Michael Stead's PhD research focuses on the environmental impacts of industrial product design in the age of ubiquitous computing, specifically the sustainability of the Internet of Things.

Paul Coulton is the Chair of Speculative and Game Design at Lancaster University's exploratory design-led research lab, Imagination.

Joseph Lindley is a postdoctoral researcher applying his doctoral research into Design Fiction as part of the PETRAS Cyber Security for the Internet of Things Research Hub. 